\title{
Manic mice turn the clock
}

DOI:

\begin{abstract}
Many circadian functions are altered in people suffering from bipolar disorder. The CLOCK protein is important in the regulation of circadian rhythms by the main body clock in the hypothalamus, and genetic studies have shown a link between variations in the CLOCK gene and symptoms associated with mania. The role of CLOCK in the aetiology of mania has long been unclear, but now Roybal et al. show that a mutation in the mouse version of CLOCK results in manic mice, and
\end{abstract}

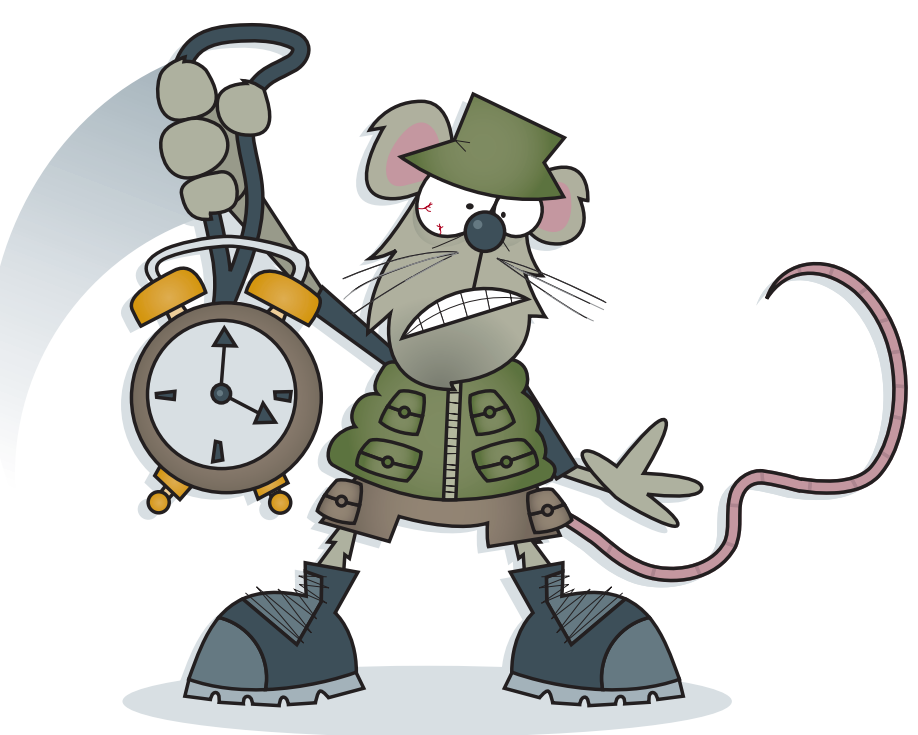

that altered CLOCK function in the ventral tegmental area (VTA) may be involved in some of the symptoms of mania.

Roybal et al. created mice in which the Clock gene carried a mutation and exposed them to a battery of behavioural tests. A self-administration paradigm, in which mice can electrically stimulate the medial forebrain bundle to induce feelings of pleasure, showed that mutant mice needed less electricity to sustain the self-administration behaviour, indicating an increased sensitivity of their reward system. Cocaine is known to increase this sensitivity, and the researchers found that it did so more in mutant than in wild-type mice. A sucrose preference test provided further evidence for an increased hedonic state in Clock mutant mice.

The Clock mutants also showed other similarities with humans in a manic state: they were less anxious and showed less learned helplessness, which has been used as an indicator of depression-like behaviour. Interestingly, a 10-day treatment with therapeutic doses of lithium, a mood stabilizer often used to treat bipolar disorder, reversed these altered behaviours to almost normal.
Because the VTA dopamine system is thought to play a part in mania, the authors next determined whether altered Clock expression specifically in this area has a role in the mania-like behaviour. They increased functional CLOCK protein levels in the VTA of the mutant mice by injecting a viral vector containing the normal Clock gene into the area. After two weeks the mice were no longer hyperactive and their reduced anxiety levels were similar to those in wild-type mice, indicating that CLOCK has an important function in regulating these behaviours.

These findings provide a greater understanding of the role of CLOCK in mania. Moreover, although the Clock mutant mice did not appear to cycle between mania and depression as in bipolar disorder, Roybal et al. have created a remarkable animal model of mania, providing an opportunity to study the mechanisms of mood stabilizer actions and to test new treatments.

Leonie Welberg

ORIGINAL RESEARCH PAPER Roybal, K. et al. Mania-like behaviour induced by disruption of CLOCK. PNAS 22 Mar 2007 (doi:10.1073/ pnas.0609625104) 